\title{
Pulse-ox paradox: potential versus pitfalls of pulse oximetry monitoring in surgical patients with obstructive sleep apnea
}

\author{
Colin M. Suen, MD, PhD • Frances Chung, MBBS
}

Received: 2 June 2019/Revised: 4 June 2019/ Accepted: 4 June 2019/Published online: 30 July 2019

(C) Canadian Anesthesiologists' Society 2019

Obstructive sleep apnea (OSA) is common in patients undergoing surgery. It is estimated that moderate to severe sleep-disordered breathing (i.e., apnea-hypopnea index $\geq$ 15) affects $14 \%$ of men and $6 \%$ of women in the general population. ${ }^{1}$ Ninety percent of patients with OSA in the general population are undiagnosed and OSA is often undiagnosed in the surgical population. ${ }^{2}$ Surgical patients are exposed to hypnotics, muscle relaxants, opioids, anxiolytics, and sedatives, all of which may worsen OSA by exacerbating upper airway collapse. Underlying mechanisms that can contribute to upper airway obstruction include suppression of the drive to breathe, depression of the arousal response, and reduced upper airway tone. Furthermore, edema associated with aggressive perioperative fluid administration may also contribute to reduced upper airway patency. In the postoperative period, $80 \%$ of death or near-death events in patients with OSA are observed within the first $24 \mathrm{hr}$

\section{M. Suen, MD, $\mathrm{PhD}$}

Department of Anesthesia, Toronto Western Hospital, University Health Network, Toronto, ON, Canada

Department of Anesthesia, University of Toronto, Toronto, ON, Canada

F. Chung, MBBS ( $\square)$

Department of Anesthesia, Toronto Western Hospital, University Health Network, Toronto, ON, Canada

e-mail: frances.chung@uhn.ca

Department of Anesthesia, University of Toronto, Toronto, ON, Canada

ResMed Chair of Anesthesia and Sleep Medicine, Department of Anesthesia and Pain Management, Toronto Western Hospital, University Health Network, University of Toronto, MCL 2-405, 399 Bathurst Rd., Toronto, ON M5T2S8, Canada after surgery with the majority of these events occurring on the hospital ward where monitoring is limited. ${ }^{3}$

While positive airway pressure (PAP) treatment remains the mainstay for patients with OSA, it is not readily available for many of those at risk of postoperative hypoxemia. Among surgical OSA patients with prescribed continuous PAP, less than 50\% are compliant. ${ }^{4}$ Furthermore, a large proportion of patients undergoing surgery have undiagnosed and untreated OSA and are at the highest risk of postoperative complications. ${ }^{5}$ The real-world scenario is less than ideal; short of PAP therapy, the default alternative on the general care ward is supplemental oxygen.

In the current issue of the Journal, Smith et al. ${ }^{6}$ report the results of a randomized, double-blind, controlled trial evaluating the potential of a peripheral transcutaneous electrical stimulation (TES) device to reduce postoperative hypoxemia in at-risk OSA patients recovering in the postanesthesia care unit (PACU). Patients at risk of OSA, based on clinical criteria, were randomized to either TES (in addition to standard PACU nurse-supported monitoring) or PACU nurse-supported monitoring (control; $n=53$ in each group). The device's closed-loop system was designed to deliver TES via an electrode placed on the patient's wrist at a pulse oximeter threshold $\mathrm{SpO}_{2} \leq$ $93 \%$. The authors show the effectiveness of the device to reduce postoperative hypoxemia during the first $60 \mathrm{~min}$ in the PACU. They report statistically significant differences between TES and control patients with regard to the primary endpoint of interest, $\mathrm{SpO}_{2}$ area under the curve $<$ $90 \%$ (median 0.0 vs $15.2 \%$ sec, $P=0.009$ ) and in the secondary endpoints, including percentage of subjects with $\mathrm{SpO}_{2}<90 \%$ (47\% vs 71\%, $P=0.03$ ), duration of $\mathrm{SpO}_{2}<$ 90\% (median 0 vs $19.1 \mathrm{sec} ; P=0.01$ ), and lowest $\mathrm{SpO}_{2}$ in the first hour $(90.5 \%$ vs $87.9 \%, P=0.04)$. 
This technology consists of an automated, closed-loop feedback system that interrupts hypoxemic episodes by TES-induced arousal that includes a compensatory ventilation response. The device relies on an ingenious algorithm that accounts for false alarm triggers secondary to movement artifacts and spurious saturation recordings. This could potentially increase the margin of safety for patients, especially for those who do not receive close monitoring once they leave the PACU (e.g., on the surgical ward). This may be particularly valuable to OSA patients with high arousal thresholds that are further exacerbated by the sedative effects of opioids. ${ }^{7}$ Such patients may experience a dangerous, vicious cycle of escalating hypoxemia to a point when the central nervous system is no longer able to mount an effective rescue arousal response, a condition known as "lights out saturation". 8

A common practice in some institutions is the "extended PACU stay", which allows for an extended (though variable) period of observation before discharge to the surgical ward. Unfortunately, the majority of complications occur after patients are discharged from the PACU and "extended PACU stays" may not be adequate. ${ }^{3}$ For postoperative surgical patients receiving opioids for analgesia, remote continuous pulse oximetry monitoring improves the detection of oxygen desaturation and is associated with decreased ICU admission rates compared with intermittent nursing spot-checks. ${ }^{9}$ Other forms of monitoring such as continuous capnography could also potentially aid in the early recognition of ventilatory abnormalities. ${ }^{9}$ In fact, the Anesthesia Patient Safety Foundation recommends that patients at increased risk for ventilation impairment, such as individuals with OSA who received perioperative opioids, should undergo both ventilation and oxygenation monitoring. ${ }^{10}$ Another recommendation is the use of continuous postoperative monitoring of ventilation and oxygenation in any patient treated with patient-controlled analgesia or neuraxial opioids. ${ }^{10}$ To date, these recommendations have not been universally adopted.

Currently, much of the evidence linking OSA to perioperative complications is in relation to nocturnal hypoxemia. ${ }^{11}$ In a recent prospective cohort study of 1,218 patients undergoing elective non-cardiac surgery, severe undiagnosed OSA was associated with a two-fold increased risk of cardiovascular complications (adjusted hazard ratio, 2.23) and longer duration of hypoxemia. ${ }^{5}$ As well, the mean cumulative duration of $\mathrm{SpO}_{2}<80 \%$ during the first three postoperative nights for patients with cardiovascular complications was increased compared with patients with no cardiovascular complications (23.1 [95\% confidence interval (CI), 15.5 to 27.7] $\mathrm{min}$ vs 10.2 [95\% CI, 7.8 to 10.9] min). In another study, we showed an association between preoperative mean $\mathrm{SpO}_{2}<93 \%$ and increased risk of postoperative complications. ${ }^{12}$

Importantly, oxygen supplementation for postoperative patients with OSA may be associated with untoward consequences. Recently, our group conducted a randomized-controlled trial of supplemental oxygen administration to postoperative patients with newly diagnosed, untreated OSA. ${ }^{13}$ Although the peripheral oxygen saturation and the apnea-hypopnea index improved, an unexpected observation was a higher proportion of hypercapnia in those treated with supplemental oxygen. Thus, the potential risk of supplemental oxygen is that a "normal" $\mathrm{SpO}_{2}$ could mask the timely recognition of hypoventilation, which may spiral into hypercarbia, $\mathrm{CO}_{2}$ narcosis, respiratory failure, and even death. ${ }^{8}$ Unfortunately, oximetry does not overcome this limitation, which highlights the pitfall of false reassurance using $\mathrm{SpO}_{2}$ monitoring. This is similar in ways to the danger of providing a high $\mathrm{F}_{1} \mathrm{O}_{2}$ using supplemental oxygen to patients with chronic obstructive pulmonary disease, which can paradoxically worsen respiratory acidosis. ${ }^{14}$ Instead, we advocate that before treating the $\mathrm{SpO}_{2}$ with supplemental oxygen, healthcare providers should interrogate the underlying cause(s) of hypoxemia and be vigilant in identifying those at risk for decompensation. The TES technology described by Smith et al. provides an exciting new means to minimize the response time between oxygen desaturation and arousal, especially if applied in general-care wards.

\section{Le paradoxe de l' 'ox' de pouls : potentiel et pièges du monitorage par oxymétrie de pouls chez les patients chirurgicaux atteints d'apnée obstructive du sommeil}

L'apnée obstructive du sommeil (AOS) est une affection répandue chez les patients subissant une chirurgie. Dans la population générale, on estime que les troubles respiratoires du sommeil modérés à sévères (soit un indice d'apnée-hypopnée $\geq 15$ ) affectent $14 \%$ des hommes et $6 \%$ des femmes. ${ }^{1}$ Quatre-vingt-dix pourcent des patients atteints d'AOS dans la population générale ne sont pas diagnostiqués et l'AOS est souvent non diagnostiquée dans la population chirurgicale. ${ }^{2}$ Les patients chirurgicaux sont exposés à des agents 
hypnotiques, des curares, des opioïdes, des agents anxiolytiques et des sédatifs - des agents pouvant tous potentiellement faire empirer une AOS en exacerbant l'affaissement des voies aériennes supérieures. Les mécanismes sous-jacents pouvant contribuer à une obstruction des voies aériennes supérieures comprennent la suppression du besoin respiratoire, la dépression de la réponse d'éveil et la réduction du tonus des voies aériennes supérieures. En outre, l'œdème associé à une administration liquidienne périopératoire significative pourrait également contribuer à réduire la perméabilité des voies aériennes supérieures. En période postopératoire, $80 \%$ des événements mortels ou quasi-mortels survenant chez des patients atteints d'AOS sont observés au cours des premières $24 \mathrm{~h}$ suivant la chirurgie, la majorité survenant à l'étage, là où le monitorage est limité. ${ }^{3}$

Bien que la ventilation par pression positive (VPP) demeure le traitement de référence pour les patients atteints d'AOS, elle n'est malheureusement pas accessible pour bon nombre des patients courant un risque d'hypoxémie postopératoire. Parmi les patients chirurgicaux atteints d'AOS auxquels une VPP continue a été prescrite, moins de $50 \%$ suivent le traitement. ${ }^{4}$ De plus, une importante proportion de patients subissant une chirurgie n'ont pas reçu de diagnostic et donc de traitement pour l'AOS et courent un risque plus élevé de complications postopératoires. ${ }^{5}$ La situation réelle est loin d'être idéale; en l'absence de traitement en VPP, l'alternative par défaut est la supplémentation d'oxygène.

Dans ce numéro du Journal, Smith et coll. ${ }^{6}$ rapportent les résultats d'une étude randomisée contrôlée à double insu évaluant le potentiel d'un stimulateur électrique transcutané (SET) périphérique pour réduire l'hypoxémie postopératoire chez les patients à risque souffrant d'AOS en salle de réveil. Les patients à risque d'AOS selon des critères cliniques ont été randomisés soit à un SET (en plus du monitorage standard par le personnel infirmier de la salle de réveil) ou au monitorage du personnel infirmier de la salle de réveil (témoin; $n=53$ dans chaque groupe). Le système en circuit fermé du stimulateur était conçu pour provoquer une stimulation électrique transcutanée via une électrode placée sur le poignet du patient à un seuil de saturométrie $\mathrm{SpO}_{2} \leq 93 \%$. Les auteurs montrent l'efficacité du stimulateur à réduire l'hypoxémie postopératoire au cours des 60 premières minutes en salle de réveil. Ils rapportent des différences statistiquement significatives entre les patients ayant reçu un SET et les patients du groupe témoin en ce qui a trait à leur critère d'évaluation principal, soit une surface sous la courbe de $\mathrm{SpO}_{2}<90 \%$ (médiane 0,0 vs $15,2 \% \mathrm{sec}, P=0,009$ ) et à leurs critères secondaires, notamment le pourcentage de patients ayant une $\mathrm{SpO}_{2}<90 \%$ (47\% vs $71 \%, P=0,03$ ), la durée de $\mathrm{SpO}_{2}<90 \%$ (médiane 0 vs $19,1 \mathrm{sec} ; P=0,01$ ) et la $\mathrm{SpO}_{2}$ la plus basse au cours de la première heure $(90,5$ $\%$ vs $87,9 \%, P=0,04)$.

Cette technologie consiste en un système de rétroaction automatisé en circuit fermé qui interrompt les épisodes d'hypoxémie par une stimulation induite par SET qui comprend une réponse ventilatoire compensatoire. Le stimulateur se fonde sur un algorithme ingénieux qui tient compte des faux déclencheurs d'alarme dus aux artéfacts de mouvement et à des évaluations erronées de la saturation. Cela pourrait potentiellement augmenter la marge de sécurité pour les patients, particulièrement pour ceux qui ne bénéficient pas d'un monitorage serré une fois qu'ils quittent la salle de réveil (par ex. à l'étage chirurgical). Cela pourrait être particulièrement utile pour les patients souffrant d'AOS et ayant des seuils de réveil très élevés qui sont d'autant plus exacerbés par les effets sédatifs des opioïdes. ${ }^{7}$ De tels patients pourraient être victimes d'un cycle dangereux et vicieux d'escalade de l'hypoxémie jusqu'au point où le système nerveux central n'est plus capable de produire de réponse de sauvetage efficace, une condition connue sous le nom de « saturation toutes lumières fermées » ('lights out saturation'). ${ }^{8}$

Le « séjour prolongé en salle de réveil » est une pratique répandue dans certaines institutions. Ainsi, on prolonge (de façon variable) la période d'observation avant de donner son congé pour l'étage de chirurgie au patient. Malheureusement, la majorité des complications surviennent après que les patients ont reçu leur congé de la salle de réveil; dès lors, des « séjours prolongés en salle de réveil » pourraient ne pas être adéquats. ${ }^{3}$ Dans le cas de patients chirurgicaux postopératoires recevant des opioïdes pour leur analgésie, un monitorage à distance et en continu de l'oxymétrie de pouls améliore le dépistage d'une désaturation en oxygène; cette mesure est associée à des taux réduits d'admission à l'USI par rapport à des contrôles intermittents par le personnel infirmier. ${ }^{9}$ D' autres formes de monitorage, tels que la capnographie en continu, pourraient également contribuer à la détection précoce des anomalies ventilatoires. ${ }^{9}$ En fait, la Fondation américaine pour la sécurité des patients en anesthésie (Anesthesia Patient Safety Foundation) recommande que les patients courant un risque accru de détérioration de la ventilation, comme par exemple les personnes atteintes d'AOS ayant reçu des opioïdes périopératoires, devraient recevoir un monitorage de la ventilation et de l'oxygénation. $^{10}$ Une autre recommandation est l'utilisation d'un monitorage postopératoire continu de la ventilation et de l'oxygénation chez tous les patients traités à l'aide d'une analgésie contrôlée par le patient ou d'opioïdes neuraxiaux. ${ }^{10}$ À ce jour, ces recommandations n'ont pas encore été adoptées de façon universelle.

À l'heure actuelle, la plupart des données probantes associant l'AOS à des complications périopératoires sont 
liées à l'hypoxémie nocturne. ${ }^{11}$ Dans une étude de cohorte prospective récente portant sur 1218 patients subissant une chirurgie non cardiaque et non urgente, une AOS grave non diagnostiquée était associée à un risque doublé de complications cardiovasculaires (rapport de risque ajusté, 2,23 ) et une durée prolongée de l'hypoxémie. ${ }^{5}$ En outre, la durée moyenne cumulée de $\mathrm{SpO}_{2}<80 \%$ pendant les trois premières nuits postopératoires chez les patients souffrant de complications cardiovasculaires était plus importante que celle des patients n'ayant pas subi de complications cardiovasculaires (23,1 [intervalle de confiance (IC) $95 \%$, 15,5 à 27,7] min vs 10,2 [IC $95 \%, 7,8$ à 10,9] min). Dans une autre étude, nous avons démontré une association entre une $\mathrm{SpO}_{2}$ moyenne préopératoire $<93 \%$ et un risque accru de complications postopératoires. ${ }^{12}$

Fait important, chez le patient postopératoire atteint d'AOS, la supplémentation en oxygène pourrait être associée à des conséquences néfastes. Notre groupe a récemment réalisé une étude randomisée contrôlée sur l'administration d'oxygène supplémentaire à des patients postopératoires ayant une AOS non traitée et nouvellement diagnostiquée. ${ }^{13}$ Bien que la saturation en oxygène périphérique et l'indice d'apnée-hypopnée se soient améliorés, une observation inattendue était la proportion plus importante d'hypercapnie chez les patients ayant reçu une supplémentation d'oxygène. Ainsi, le risque potentiel de la supplémentation en oxygène est qu'une $\mathrm{SpO}_{2}$ «normale » puisse entraver le dépistage en temps opportun d'une hypoventilation, laquelle pourrait se détériorer en hypercarbie, en narcose au $\mathrm{CO}_{2}$, en insuffisance respiratoire, voire en décès. ${ }^{8}$ Malheureusement, l'oxymétrie n'est pas capable de surmonter cet obstacle, ce qui souligne le piège de fausse rassurance que tend le monitorage de la $\mathrm{SpO}_{2}$. D'une certaine manière, cela ressemble au danger de procurer une $\mathrm{F}_{1} \mathrm{O}_{2}$ élevée à l'aide d'oxygène supplémentaire chez les patients atteints de maladie pulmonaire obstructive chronique, une mesure qui pourrait, de façon paradoxale, empirer l'acidose respiratoire. ${ }^{14}$ Nous préconisons plutôt, avant de traiter la $\mathrm{SpO}_{2}$ à l'aide d'oxygène supplémentaire, que les fournisseurs de soins de santé s'interrogent sur la ou les causes sous-jacentes de l'hypoxémie et qu'ils soient prudents dans leur identification des personnes à risque de décompensation. La technologie de SET décrite par Smith et coll. offre une nouvelle modalité intéressante pour minimiser le temps de réaction entre une désaturation en oxygène et le réveil, particulièrement si elle est appliquée dans les départements de soins généraux.

Disclosures Frances Chung has received research support from the Ontario Ministry of Health and Long-Term Care, University Health Network Foundation, Acacia Pharma, Medtronics grants to institution outside of the submitted work, Up-to-date royalties, and STOP-Bang proprietary to University Health Network.
Conflicts of interest None declared.

Editorial responsibility This submission was handled by Dr. Steven Backman, Associate Editor, Canadian Journal of Anesthesia.

Déclarations Frances Chung a reçu un soutien à la recherche par le biais de bourses du ministère de la Santé et des Soins de longue durée de l'Ontario, de la Fondation de l'University Health Network, d'Acacia Pharma, de Medtronics à son institution en dehors de ce manuscrit, des droits d'auteur de Up-to-date, et de STOP-Bang, breveté par le University Health Network.

\section{Conflit d'intérêt Aucun.}

Responsabilit' éditoriale Cet article a été traité par Dr Steven Backman, rédacteur adjoint, Journal canadien d'anesthésie.

\section{References}

1. Peppard PE, Young T, Barnet JH, Palta M, Hagen EW, Hla KM. Increased prevalence of sleep-disordered breathing in adults. Am J Epidemiol 2013; 177: 1006-14.

2. Singh M, Liao P, Kobah S, Wijeysundera DN, Shapiro C, Chung $F$. Proportion of surgical patients with undiagnosed obstructive sleep apnoea. Br J Anaesth 2013; 110: 629-36.

3. Subramani $Y$, Nagappa M, Wong J, Patra J, Chung F. Death or near-death in patients with obstructive sleep apnoea: a compendium of case reports of critical complications. Br J Anaesth 2017; 119: 885-99.

4. Mehta V, Subramanyam R, Shapiro CM, Chung F. Health effects of identifying patients with undiagnosed obstructive sleep apnea in the preoperative clinic: a follow-up study. Can J Anesth 2012; 59: 544-55.

5. Chan MT, Wang CY, Seet E, et al. Association of unrecognized obstructive sleep apnea with postoperative cardiovascular events in patients undergoing major noncardiac surgery. JAMA 2019; 321: 1788-98.

6. Smith HM, Kilger J, Burkle CM, Schroeder DR, Gali B. Peripheral electrical stimulation reduces postoperative hypoxemia in patients at risk for obstructive sleep apnea: a randomized-controlled trial. Can J Anesth 2019; 66: DOI: https:// doi.org/10.1007/s12630-019-01451-3.

7. Subramani Y, Singh M, Wong J, Kushida CA, Malhotra A, Chung $F$. Understanding phenotypes of obstructive sleep apnea: applications in anesthesia, surgery, and perioperative medicine. Anesth Analg 2017; 124: 179-91.

8. Lynn LA, Curry JP. Patterns of unexpected in-hospital deaths: a root cause analysis. Patient Saf Surg 2011; 5: 3.

9. Lam T, Nagappa $M$, Wong $J$, Singh $M$, Wong D, Chung $F$. Continuous pulse oximetry and capnography monitoring for postoperative respiratory depression and adverse events: a systematic review and meta-analysis. Anesth Analg 2017; 125: 2019-29.

10. Frederickson TW, Lambrecht JE. Using the 2018 Guidelines from the Joint Commission to Kickstart Your Hospital's Program to Reduce Opioid-Induced Ventilatory Impairment. APSF Newsletter; 2018: 33:1. Available from URL: https://www.apsf. org/article/using-the-2018-guidelines-from-the-joint-commissionto-kickstart-your-hospitals-program-to-reduce-opioid-inducedventilatory-impairment/ (accessed June 2019). 
11. Suen C, Ryan CM, Mubashir T, et al. Sleep study and oximetry parameters for predicting postoperative complications in patients with OSA. Chest 2019; 155: 855-67.

12. Chung F, Zhou L, Liao P. Parameters from preoperative overnight oximetry predict postoperative adverse events. Minerva Anestesiol 2014; 80: 1084-95.

13. Liao P, Wong J, Singh $M$, et al. Postoperative oxygen therapy in patients with OSA: a randomized controlled trial. Chest 2017; 151: 597-611.
14. Agusti AG, Carrera M, Barbe F, Munoz A, Togores B. Oxygen therapy during exacerbations of chronic obstructive pulmonary disease. Eur Respir J 1999; 14: 934-9.

Publisher's Note Springer Nature remains neutral with regard to jurisdictional claims in published maps and institutional affiliations. 